\title{
Sidestream dark field images of the microcirculation: intra-observer reliability and correlation between two semi-quantitative methods for determining flow
}

\author{
Sandra M Petersen ${ }^{*}$, Gorm Greisen, Simon Hyttel-Sorensen and Gitte H Hahn
}

\begin{abstract}
Background: Since analysis of Sidestream Dark Field images still requires subjective interpretation, we wanted to determine intra-observer repeatability and to estimate the correlation between different evaluation methods.

Methods: Fifty-four Sidestream Dark Field videos were analyzed twice by the same blinded observer using validated software. Vessels were detected, generating the parameter Total Vessel Density (TVD), and flow was determined by (i) classifying each vessel separately, generating the parameters Perfused Vessel Density (PVD) and Proportion of Perfused Vessels (PPV), and by (ii) the "Boerma" method, generating a Microvascular Flow Index (MFI) by quadrants.

Results: Intraclass Correlation Coefficients (ICCS) were above 0.9 for TVD and above 0.8 for PDV and PPV. $\mathrm{MFI}_{\text {by }}$ quadrants had the lowest reliability (ICC $=0.52$ for capillaries and ICC $=0.59$ for all vessels), significantly lower than for PVD (ICC $=0.89, p<0.001$ for capillaries and ICC $=0.90, p<0.001$ for all vessels) and PPV (ICC $=0.82$, $p=0.003$ for capillaries and ICC $=0.83, p=0.01$ for all vessels). Correlation coefficient ( $r$ ) between PPV and $\mathrm{MFI}_{\text {by quadrants }}$ corrected for measurement error was $0.39(0.10-0.64)$ for capillaries and $1.01(0.85-1.16)$ for all vessels.

Conclusions: Intra-observer reliability for full evaluation of Sidestream Dark Field images was good for vessel detection and for flow classification but significantly poorer for the faster "Boerma" method. Furthermore, the Boerma method is likely to estimate different aspects of capillary flow than do the standard methods.
\end{abstract}

Keywords: Videomicroscopy, Microcirculation, Sidestream dark field, Total vessel density, Perfused vessel density, Microvascular flow index, Proportion of perfused vessels, Reliability

\section{Background}

The microscopic techniques Orthogonal Polarization Spectral (OPS) imaging [1] and is successor Sidestream Dark Field (SDF) imaging [2] enable visualization of the microcirculation. The technique is available as a hand held device. It allows bedside investigation of the microcirculation in multiple kinds of tissue and is applied in both clinical $[3,4]$ and experimental [5-7] research. It is argued to be the best method to evaluate heterogeneity of microcirculatory flow [8].

\footnotetext{
* Correspondence: sandrameinich@gmail.com

Department of Neonatology, Copenhagen National Hospital, Rigshospitalet, Copenhagen DK-2100, Denmark
}

(C) 2014 Petersen et al.; licensee BioMed Central Ltd. This is an Open Access article distributed under the terms of the Creative Commons Attribution License (http://creativecommons.org/licenses/by/2.0), which permits unrestricted use, distribution, and reproduction in any medium, provided the original work is properly credited.
Software packages have been developed to evaluate SDF images semi-automatically [9]. This software detects vessels, but human interaction is still required to delete incorrectly detected vessels and to trace undetected vessels manually. Flow determination cannot yet be done automatically. It requires a semi-quantitative analysis involving subjective assessment. Two methods for flow determination are predominant: 1 ) the "per vessel" method, where flow in each vessel is classified separately and 2) the "Boerma" method, where the image is split into four quadrants and the predominant type of flow in each quadrant is determined.

The aim of present study was (i) to determine intraobserver agreement of vessel detection and flow classification 
by both the "per vessel" and the "Boerma" method; and (ii) to examine the correlation between the two methods.

\section{Methods}

\section{Ethics}

The animals were anaesthetized with Propofol during the entire experiment and the Danish Animal Experiments Inspectorate approved the experimental protocol (approval ID: 2009/561-1723).

\section{Animals}

Sidestream Dark Field (SDF) videos of the cerebral cortex were recorded by one operator (G.H.H.) through a parietal craniotomy (diameter: $15 \mathrm{~mm}$ ) in 12 newborn piglets (median age: 1 day, range: 1-6 days). Dura was removed carefully, avoiding damage to the cortex. Bleeding from the bone was avoided by Bone $\mathrm{Wax}^{\mathrm{TM}}$. During the experiment, which lasted approximately 5 hours, the cortical area was covered by wet gauzes. To prevent drying of the examined area, $2 \mathrm{ml}$ saline solution $\left(37^{\circ} \mathrm{C}\right)$ was administered every 15 minutes.

The videos were obtained as a part of a larger experimental study of cerebral blood flow published elsewhere [10]. In short the animals were exposed to blood pressure elevations of $10 \mathrm{mmHg}$ by means of epinephrine, norepinephrine and dopamine. Each drug was compared to a reference method, thus giving six video sessions in each piglet.

\section{Sidestream dark field (SDF)}

The SDF videos were obtained with a video microscope from Microscan, MicroVision Medical Inc., Amsterdam, Netherlands. The video microscope illuminates $1.08 \times$ $0.81 \mathrm{~mm}$ and penetrates $0.9 \mathrm{~mm}$ with light in a wavelength $(580 \mathrm{~nm})$ that is absorbed by red blood cells (RBC). The RBCs therefore appear dark, and the viewer can trace the movement of each $\mathrm{RBC}$. This gives information of the architecture of the microvasculature and erythrocyte flow in each vessel.

Image acquisition was performed according to published consensus criteria [11]. In practice, the lens was applied directly to the cerebral cortex. Adequate focus was found and in order to avoid pressure artifacts, pressure from the device was released slowly until flow could be seen in all large vessels. The videos were recorded with a length of $25 \mathrm{~s}$ each. Each video clip was stored as an AVI-file to allow computerized frame by frame analysis. For each session (six in total in each piglet), recordings that were out of focus or not steady enough were discarded and a new recording was made, until three videos were stored. Thus, we ended up with a total of 18 videos in each piglet.

\section{Experimental protocol}

In the first run, the videos were given a code each, and blinded evaluation was performed on video recordings from all 12 piglets $(n=216)$ by one investigator (S.M.P.) who performed all steps in the analysis after the recording was made. In the second run, 3 of the 12 piglets were chosen at random, the videos $(n=54)$ were recoded, and blinded analysis was performed once again by the same investigator. The present study is based on the double analysis of these 54 videos." Run 1 " represents the data generated from the first evaluation and "run 2" represents the data generated from the second evaluation.

Analysis of the videos was performed using the validated software package Automated Vascular Analysis; AVA 3.0 from Micro Vision Medical Inc., Amsterdam, Netherlands [9]. A stable video fragment as long as possible was selected for further analysis, the video was corrected for background variation and the image contrast was optimized. After detecting vessels automatically, incorrectly detected vessels were deleted and undetected vessels were drawn manually or using the detection tool. Wrongly disconnected segments were chained and wrongly connected segments were unchained.

Velocity classification was performed by eye: (i) "per vessel" and (ii) with the "Boerma" method [11]. Flow was categorized as: "no flow", "intermittent flow" (50\% of the time with no flow), "sluggish flow" (slow but continuous) or "continuous flow". To score the images with the "Boerma" method, the video was split into four quadrants, and the predominant flow of small, medium, large and very large vessels was classified in each of these quadrants. Vessels with a diameter $<20 \mu \mathrm{m}$ was termed small [11]. In the following, these small vessels will be referred to as capillaries.

\section{Consensus parameters}

An analysis report with the consensus parameters was created by the software program [12]. The parameter Total Vessel Density (TVD) $\left(\mathrm{mm} / \mathrm{mm}^{2}\right)$ reflects vessel detection. The parameters Perfused Vessel Density (PVD) $\left(\mathrm{mm} / \mathrm{mm}^{2}\right)$ and Proportion of Perfused Vessels (PPV) (\%) reflect the "per vessel" method for evaluating flow. Perfused vessels were defined as all vessels classified as "continuous" or "sluggish" [11]. PPV was calculated by the software as the percentage of total vessel length that was perfused. The "Boerma" method for flow classification generated a Microvascular Flow Index by quadrants ( MFI $_{\text {by quadrants }}$ ) which was calculated by evaluating the overall flow in each quadrant (no flow $=0$, intermittent $=1$, sluggish $=2$, continuous $=3$ ), and then averaging the score over the four quadrants [13].

When re-analyzing the videos, the investigator started once again with the "raw" version of the video, meaning 
that all steps in the analysis were repeated, from choosing a stable fragment of the video to classifying the flow of the vessels.

The repeatability of SDF-video analysis was assessed by the repeatability of the consensus parameters for both capillaries (c) and for all vessels (a) as recommended [11].

\section{Statistics}

Intra-observer repeatability was determined for each of the eight consensus parameters. The distribution of some of the parameters was not normal, thus both parametric and non-parametric methods were used. The results did not differ substantially, thus only results of parametric analysis are presented. Paired $\mathrm{t}$-test was performed to compare data from run 1 and run 2. The Bland and Altman method was used to estimate agreement [14]. To assess reliability, intraclass correlation coefficients (ICCs) were calculated from a two way random effects model with absolute agreement for single measures. Since both PPV and $M I_{\text {by quadrants }}$ are prone to measurement error, i. e., unexplained variability, the estimated correlation coefficient between the measures will be biased towards zero. This regression dilution was corrected by the Spearman correction for attenuation [15]. Tools from www.vassarstats.net were used to calculate the significance of the difference between ICC's and the confidence intervals of the correlation coefficients between PPV and MFI $_{\text {by quadrants, }}$ both with the Fischer r-to-z transformation. All other calculations were made using the program IBM SPSS statistics 20. A two tailed p-value below 0.05 was considered significant.

\section{Results}

All 54 videos were fully evaluated and results for the eight consensus parameters were calculated for all 108 evaluations. There was a tendency towards an increase in mean TVD and mean PVD from run 1 to run 2, but only statistically significant for TVDa $(p=0.01)$. PPV did not change from run 1 to run 2 whereas MFI-scoring decreased significantly from run 1 to run 2 (MFIc by qaudrants: $\mathrm{p}=0.01$ and MFIa by quadrants: $\mathrm{p}=0.03$ ) (Table 1$)$. The agreement for all 8 parameters (Table 1) was inspected visually in Bland Altman plots. One outlier was seen for PVD, PPV and $\mathrm{MFI}_{\text {by quadrants, }}$ but there was no sign of heteroscedasticity for any of the eight parameters. Perfused vessel density for capillaries is shown as an example (Figure 1).

The ICC was higher than 0.5 for all 8 parameters (Table 2). TVD, the parameters reflecting vessel detection, had the highest reliability with an ICC of 0.94 for capillaries and 0.92 for all vessels. Regarding flow classification, $\mathrm{MFI}_{\text {by quadrants }}$ had the lowest reliability (ICC $=0.52$ for capillaries and ICC $=0.59$ for all vessels), significantly lower than for PVD ( $\mathrm{ICC}=0.89, \mathrm{p}<0.001$ for capillaries and $\mathrm{ICC}=0.90, \mathrm{p}<0.001$ for all vessels $)$ and PPV $(\mathrm{ICC}=0.82$, $\mathrm{p}=0.003$ for capillaries and ICC $=0.83, \mathrm{p}=0.01$ for all vessels).

The correlation between $\mathrm{MFI}_{\text {by quadrants }}$ and PPV was visualized in scatter plots (Figure 2). Correlation coefficient ( $\mathrm{r}$ ) between PPV and $\mathrm{MFI}_{\text {by }}$ quadrants was 0.25 $(0.066-0.42)$ for capillaries and $0.71(0.60-0.81)$ for all vessels. After the Spearman correction for attenuation the correlation coefficient was $0.39(0.10-0.64)$ for capillaries and $1.01(0.85-1.16)$ for all vessels.

\section{Discussion}

This blinded intra-observer study shows that the repeatability for semi-quantitative analysis of SDF imaging is good for both vessel detection and flow classification with reliability above 0.9 for vessel detection (TVD) and above 0.8 for flow evaluation using the "per vessel" method (PVD and PPV). For the "Boerma" method $\left(\mathrm{MFI}_{\text {by }}\right.$ quadrants $)$, however, reliability was relatively weak. Furthermore, the correlation between PPV and

Table 1 Mean and (SD), including mean difference and limits of agreement (LA)

\begin{tabular}{lllll}
\hline & Run $\mathbf{1}(\mathbf{n}=\mathbf{5 4})$ & Run $\mathbf{2}(\mathbf{n}=\mathbf{5 4})$ & $\mathbf{p}$ & Mean difference (LA) \\
\hline Capillaries & & & & \\
TVD & $13.1(3.3)$ & $13.4(3.3)$ & 0.06 & $-0.31(-2.62$ to 2.00$)$ \\
PVD & $11.8(3.0)$ & $12.2(3.2)$ & 0.07 & $-0.36(-3.20$ to 2.47$)$ \\
PPV & $65.1(10.7)$ & $65.8(11.3)$ & 0.42 & $-0.74(-14.1$ to 12.6$)$ \\
MFI & $2.7(0.3)$ & $2.6(0.4)$ & 0.01 & $0.12(-0.52$ to 0.77$)$ \\
All vessels & & & $-0.37(-2.53$ to 1.79$)$ \\
TVD & $16.7(2.9)$ & $17.0(2.9)$ & 0.01 & $-0.31(-2.92$ to 2.29$)$ \\
PVD & $14.5(2.9)$ & $14.8(3.0)$ & 0.08 & $-0.03(-10.6$ to 10.5$)$ \\
PPV & $87.0(8.1)$ & $87.0(9.8)$ & 0.97 & 0.03 \\
MFI & $2.8(0.2)$ & $2.7(0.3)$ & $0.08(-0.41$ to 0.56$)$ \\
\hline
\end{tabular}

Data is shown for Total Vessel Density (TVD, $\mathrm{mm} / \mathrm{mm}^{2}$ ), Perfused Vessel Density (PVD, $\mathrm{mm} / \mathrm{mm}^{2}$ ), Proportion of Perfused Vessels (PPV, \%) and Microvascular Flow Index (MFI). 


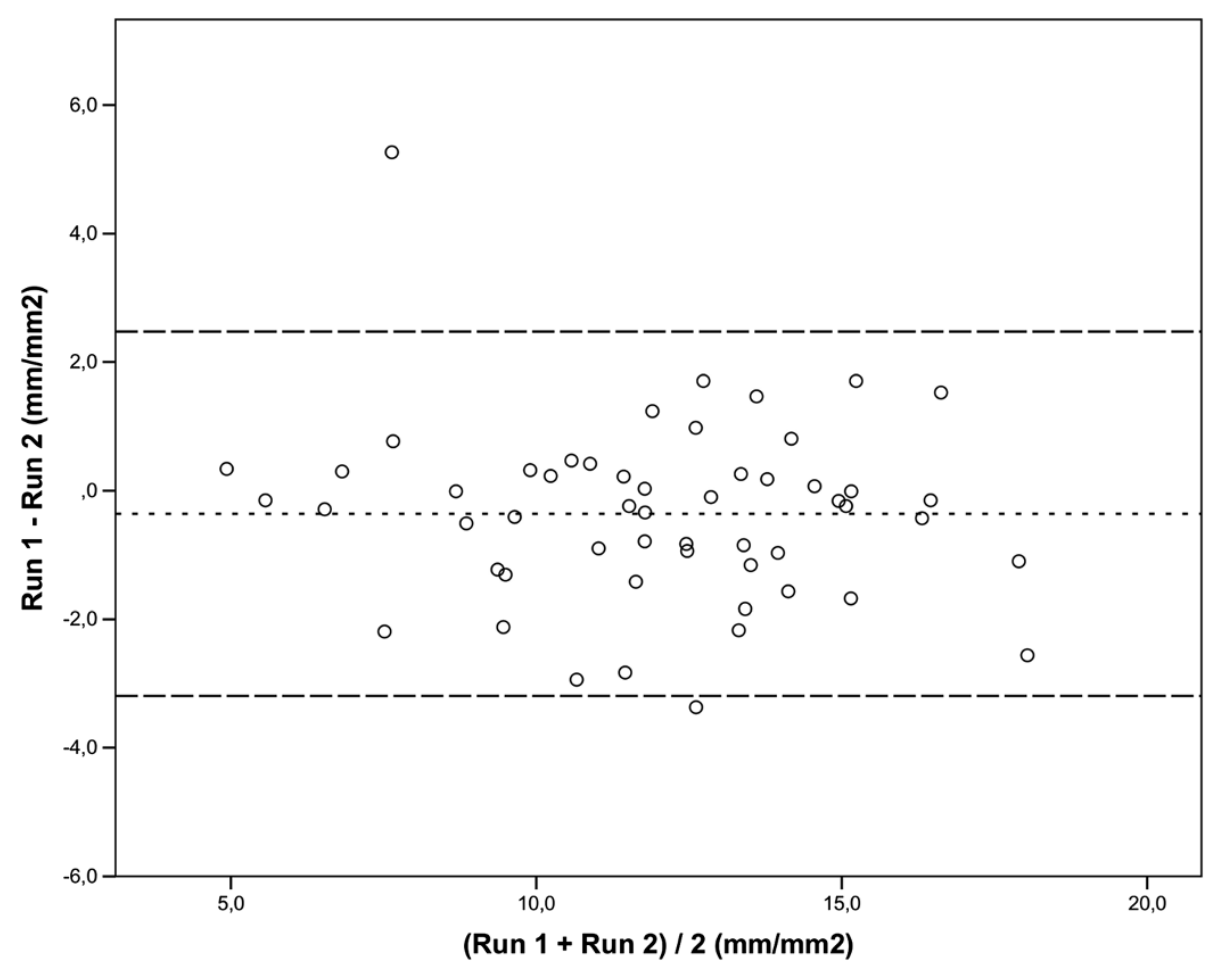

Figure 1 Bland and Altman plot for capillary Perfused Vessel Density. Figure showing bias (...) and limits of agreement (mean $+/-2$ SD) (---).

$\mathrm{MFI}_{\text {by quadrants }}$ was low for capillaries but almost perfect for all vessels after correcting for the measurement error found in this study.

The strengths of this study are: (i) We studied anaesthetized piglets, and the only movement artifact will be that of the investigator holding the camera which is a realistic setting for most animal studies. (ii) Image acquisition was performed as recommended in a published round table report to minimize movement artifacts,

Table 2 Intraclass Correlation Coefficient (ICC) and 95\% Confidence interval $(\mathrm{CI})$

\begin{tabular}{ll}
\hline Capillaries & ICC (95\% Cl) \\
TVD & $0.94(0.89-0.96)$ \\
PVD & $0.89(0.82-0.94)$ \\
PPV & $0.82(0.70-0.90)$ \\
MFI by quadrants & $0.52(0.29-0.70)$ \\
All vessels & \\
TVD & $0.92(0.86-0.98)$ \\
PVD & $0.90(0.83-0.97)$ \\
PPV & $0.83(0.72-0.90)$ \\
MFI by quadrants & $0.59(0.38-0.74)$ \\
\hline
\end{tabular}

Data is shown for Total Vessel density (TVD), Perfused Vessel Density (PVD), Proportion of Perfused Vessels (PPV) and Microvascular Flow Index by quadrants ( $\mathrm{MFI}_{\text {by quadrants }}$ ). pressure artifacts and focus problems [11]. (iii) The investigator evaluating the videos was not involved in the process of acquiring images and the evaluations were performed blinded keeping bias at a minimum. (iv) An instruction was made before analyzing the videos to make sure that each video was evaluated in a standardized manner. This instruction was based on the manual and on experiences from an evaluation performed on a random video by two of the investigators (S.M.P. and G. H.H.). Finally, (v) the investigator analyzed a total of 216 videos before reanalyzing 54 of them again for this study, making it impossible to remember the videos from run 1 when presented for them again in run 2 . The small drift in the mean value of some of the parameters from run 1 to run 2 shows that the standardization was effective although not perfect.

This study has limitations as well: (i) The investigated tissue was cerebral cortex depleted of dura and cerebrospinal fluid for several hours, thus the visualized microcirculation and quality of the images might have been affected. (ii) Image quality might also have been affected by the experimental setup, where the piglets were exposed to several induced blood-pressure elevations. (iii) Finally, due to movement artifacts the stabilized videos became shorter than 20 seconds in some of the videos, and in these cases classification of vessels had to be based on shorter fragments than recommended. 

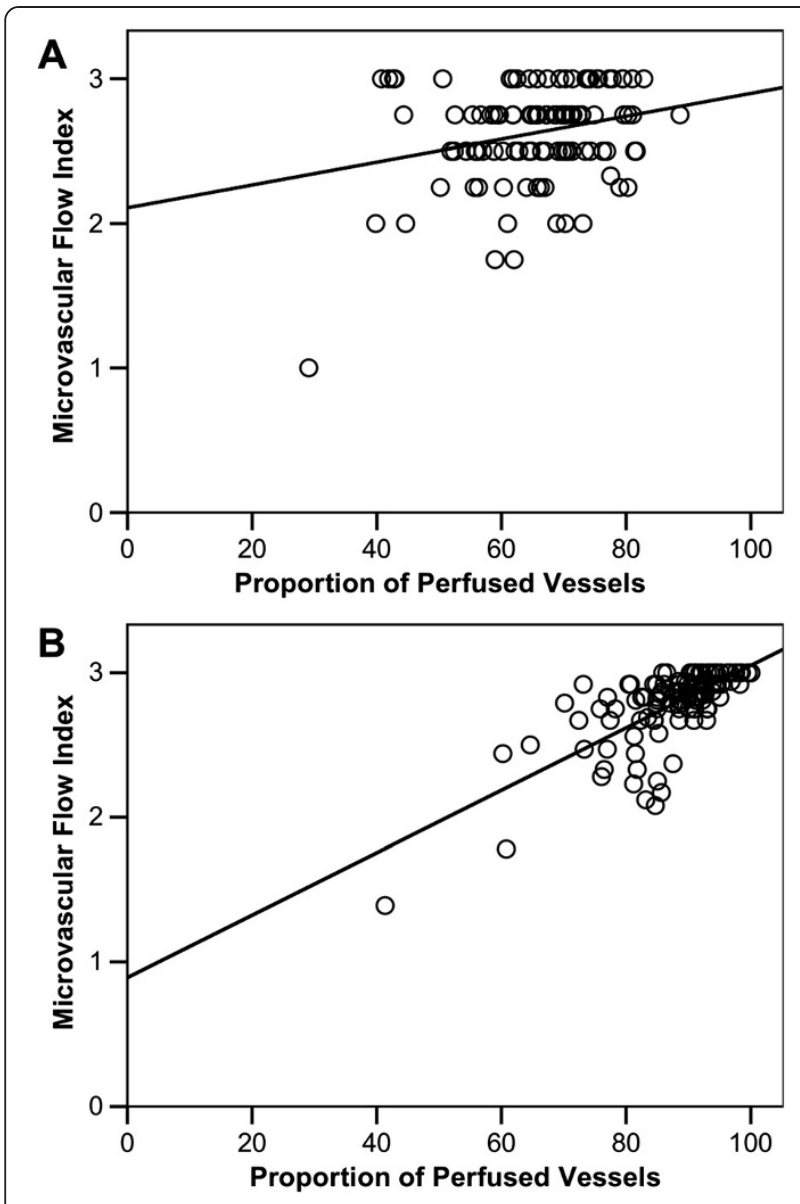

Figure 2 Correlation between Proportion of Perfused Vessels and Microvascular Flow Index by quadrants. Correlation is shown for capillaries (A) and for all vessels (B).

We reported a low reliability of the $\mathrm{MFI}_{\text {by quadrants. In }}$ another study, the "Boerma" method was validated using kappa statistics, and high kappa values for both intraand inter-observer variability were reported (kappa = 0.75 and 0.85 respectively) [13]. In our study, the mean $\mathrm{MFI}_{\text {by quadrants }}$ was high $(2.6-2.8)$, because most of the quadrants were given the classification 3 for continuous flow (Figure 2), whereas in the validation study the classification of quadrants was more equally distributed between 0 and 3 , and a mean of 2.2 for sublingual site classifications and 1.8 for stoma site classifications could be calculated from the published data. This will of course influence the reliability of the method, since videos with high between-subject variability are easier to distinguish than videos with less variability. Apart from that it might reflect that it is easier to distinguish between intermittent and sluggish flow (1 and 2) than between sluggish and continuous flow (2 and 3 ).

Recently, the "Boerma" method was compared to a method where every vessel was classified, and a parameter called $\mathrm{MFI}_{\mathrm{vessel}}$ by vessel was generated as the mean of all the classifications [16]. SDF images were evaluated with both methods, and the agreement between them was poor (bias $+/$ - precision $0.21 \pm 0.73, P=0.05$ ). The

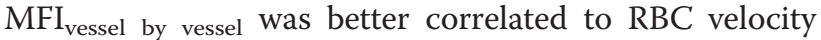
(calculated using space time diagrams) and to the PPV, than the $\mathrm{MFI}_{\text {by quadrants. This supports the findings of }}$ our study suggesting that a more reliable evaluation of the images requires classification of each vessel separately.

In our study, we calculated the correlation between PPV and MFI $_{\text {by quadrants. Low correlation was found for }}$ capillaries, even after correction for measurement error. A low correlation was also found between PPV and $\mathrm{MFI}_{\text {by quadrants }}\left(\mathrm{R}^{2}=0.54, \mathrm{P}=0.0001\right)$ in the study concerned with different kinds of MFI's [16], but in a recent study of patients with sepsis a stronger correlation for capillaries was found $\left(\mathrm{R}^{2}=0.92, \mathrm{p}=0.0001\right)$ [17]. However, none of the studies corrected for measurement error. Both studies used videos obtained from critically ill patients, and MFIs were more equally distributed between values of 0 and 3 than in our study. Based on these correlations it cannot be concluded whether or not PPV and MFI evaluate capillary flow in the same way. Furthermore, we found a correlation above 1 for all vessels. This might indicate an underestimate of the reliability of the methods [18].

The "Boerma" method has some general problems apart from the lack of reliability. It does not differ between very heterogeneous flow and generally homogenous, but sluggish flow. As an example, if flow is very heterogeneous, two quadrants might be classified as having continuous flow, and the other two as having intermittent flow (3, 3, 1 and 1). This yields an $\mathrm{MFI}_{\text {by quadrants }}$ of 2 , as would also be generated if all four quadrants were classified as having sluggish flow (2, 2, 2 and 2). Thus, two very different situations will be interpreted as the same using the $\mathrm{MFI}_{\text {by quadrants. }}$ Furthermore, MFI is a categorical parameter and an increase from 1 to 2 does not necessarily imply the same amount of change in perfusion as an increase from 2 to 3 . Further studies are needed to establish if this could be part of the explanation of the low correlation found between PPV and $\mathrm{MFI}_{\text {by quadrants }}$ for capillaries in our study. The high correlation for all vessels might be explained by larger vessels being easier to classify than the small capillaries.

The "Boerma" method also has some advantages compared to the "per vessel" method. Classifying each vessel separately is a time-consuming process and evaluating a video file of 20 seconds duration with the "per vessel" method takes between 10 and 45 minutes depending on number of capillaries in the area, the contrast of the images, movement-artifacts and bleeding in the tissue. With a modification of the "Boerma" method a real-time, point of care evaluation can be made in two minutes [19]. New 
rapid automated methods for vessel density assessment has been developed but automated velocity assessment still has many limitations, especially if heterogeneity is to be assessed [20,21]. If SDF imaging is used in research, an evaluation time of approximately 30 minutes per video will be acceptable, but if it is supposed to become a clinical tool, evaluation time has to be short. This represents the main advantage for the" Boerma" method.

The remaining question is, if the parameters generated from the analysis of SDF images provide information that can't be found using less time consuming techniques. As laser Doppler technique can provide information of changes in blood flow [22], bedside assessment of heterogeneity remains the most promising new aspect of SDF. Clinical studies using SDF have demonstrated increased microcirculatory heterogeneity in patients with sepsis compared to healthy volunteers, and larger changes among non-survivors than survivors $[17,23]$. As described earlier, the MFI does not provide information regarding heterogeneity, neither does PPV. A heterogeneity index has been proposed where images recorded from three to five sites are analyzed, and highest minus lowest site MFI divided by mean MFI of the sites are calculated [23]. Low reliability of MFI and the natural oscillations of the vasculature over time [24] taken into account, this method may not be able to detect small changes in heterogeneity. In that respect PPV seems as the superior method for evaluation of microcirculatory heterogeneity.

Does the quality of image acquisition affect the analysis of the images? In our study one major outlier serves as an example of how image acquisition problems can affect later evaluation. The outlier was detected for all velocity parameters when inspecting the Bland Altman plots (Figure 1). The video-file was inspected and revealed a constant slight movement of the camera in the 20 second long sequence. Comparison of the two different stabilized video-files generated from run 1 and 2 showed that two different parts of the video was selected for stabilization. This means that the rest of the analysis was made on two different parts of the video. Therefore video recordings with good quality are essential. The hand held device might not enable such high quality recordings [25]. An image acquisition stabilizer has been developed, but is not available for clinical use [26].

\section{Conclusions}

In conclusion, this study demonstrated that intraobserver repeatability for evaluation of SDF images was good for vessel detection (TVD) and for flow classification, when each vessel was classified separately (PVD and PPV). A significantly poorer repeatability was found for the "Boerma" method and PPV therefore seems as the superior method for evaluation of microcirculatory heterogeneity, especially when detecting small changes. Furthermore, correlation between the $\mathrm{MFI}_{\text {by quadrants }}$ and PPV after correcting for measurement error was low for capillaries but almost perfect for all vessels. Thus, it seems that the Boerma method quantifies different aspects of capillary flow than does PPV.

\section{Abbreviations \\ SDF: Sidestream dark field; TVD: Total vessel density; PVD: Perfused vessel density; PPV: Proportion of perfused vessels; MFI: Microvascular flow index; c: For capillaries; a: For all vessels; ICC: Intraclass Correlation Coefficient.}

\section{Competing interests}

All authors declare that they have no competing interests.

\section{Authors' contributions}

SMP made all analyses of the SDF images, conducted the statistical analyses and drafted the manuscript. GHH and SHS conducted the piglet studies and $\mathrm{GHH}$ recorded the SDF images. GG supervised the statistical analysis. GG and $\mathrm{GHH}$ conceived of this study and supervised the drafting of the manuscript. All authors revised the manuscript for important intellectual content and approved the final draft.

\section{Acknowledgements}

We would like to thank Ole Pryds (MD, DMSci) for skillful guidance during the experiments.

\section{Financial support}

This study was supported by the Lundbeck Foundation, the Aase and Einar Danielsen Foundation and the Augustinus Foundation.

Received: 30 July 2013 Accepted: 9 April 2014

Published: 6 May 2014

\section{Reference}

1. Groner W, Winkelman JW, Harris AG, Ince C, Bouma GJ, Messmer K, Nadeau RD: Orthogonal polarization spectral imaging: a new method for study of the microcirculation. Nat Med 1999, 5:1209-1212.

2. Goedhart PT, Khalilzada M, Bezemer R, Merza J, Ince C: Sidestream Dark Field (SDF) imaging: a novel stroboscopic LED ring-based imaging modality for clinical assessment of the microcirculation. Opt Express 2007, 15:15101-15114.

3. Vincent $J \mathrm{~L}$, De BD: Microcirculatory alterations in the critically ill. Hosp Pract (Minneap) 2009, 37:107-112.

4. Ince C: The microcirculation is the motor of sepsis. Crit Care 2005, 9(Suppl 4):S13-S19.

5. Yeh YC, Sun WZ, Ko WJ, Chan WS, Fan SZ, Tsai JC, Lin TY: Dexmedetomidine prevents alterations of intestinal microcirculation that are induced by surgical stress and pain in a novel rat model. Anesth Analg 2012, 115:46-53.

6. Taccone FS, Su F, Pierrakos C, He X, James S, Dewitte O, Vincent $J$, De Backer D: Cerebral microcirculation is impaired during sepsis: an experimental study. Crit Care 2010, 14:R140.

7. Milstein DM, Lindeboom JA, Ince C: Intravital sidestream dark-field (SDF) imaging is used in a rabbit model for continuous noninvasive monitoring and quantification of mucosal capillary regeneration during wound healing in the oral cavity: a pilot study. Arch Oral Biol 2010, 55:343-349.

8. De BD, Donadello K, Cortes DO: Monitoring the microcirculation. J Clin Monit Comput 2012, 26:361-366.

9. Dobbe JG, Streekstra GJ, Atasever B, Van ZR, Ince C: Measurement of functional microcirculatory geometry and velocity distributions using automated image analysis. Med Biol Eng Comput 2008, 46:659-670.

10. Hahn GH, Hyttel-Sorensen S, Petersen SM, Pryds O, Greisen G: Cerebral effects of commonly used vasopressor-inotropes: a study in newborn piglets. PLoS One 2013, 8:e63069.

11. De Backer D, Hollenberg S, Boerma C, Goedhart P, Buchele G, Ospina-Tascon G, Dobbe I, Ince C: How to evaluate the microcirculation: report of a round table conference. Crit Care 2007, 11:R101. 
12. Dobbe JG: Automated Vascular Analysis version 3.0. Academic Medical Center, University of Amsterdam: Dept. Medical Technological Development (MTO); 2007

13. Boerma EC, Mathura KR, van der Voort PH, Spronk PE, Ince C: Quantifying bedside-derived imaging of microcirculatory abnormalities in septic patients: a prospective validation study. Crit Care 2005, 9:R601-R606.

14. Bland JM, Altman DG: Statistical methods for assessing agreement between two methods of clinical measurement. Lancet 1986, 1:307-310.

15. Kromrey JD, Fay RH, Bellara AD: ATTENUATE: A SAS ${ }^{\circledR}$ Macro for Computing Confidence Intervals for Disattenuated Correlation Coefficients. Tampa, FL: University of South Florida; 2008. http://analytics.ncsu.edu/sesug/2008/PO089.pdf. Ref Type: Online Source.

16. Pozo MO, Kanoore Edul VS, Ince C, Dubin A: Comparison of different methods for the calculation of the microvascular flow index. Crit Care Res Pract 2012, 2012:102483.

17. Edul VS, Enrico C, Laviolle B, Vazquez AR, Ince C, Dubin A: Quantitative assessment of the microcirculation in healthy volunteers and in patients with septic shock. Crit Care Med 2012, 40:1443-1448.

18. Winne PH, Belfry MJ: Interpretive problems when correcting for attenuation. J Educ Meas 1982, 19:125-134.

19. Arnold RC, Parrillo JE, Phillip DR, Chansky ME, Shapiro NI, Lundy DJ, Trzeciak S, Hollenberg SM: Point-of-care assessment of microvascular blood flow in critically ill patients. Intensive Care Med 2009, 35:1761-1766.

20. Bezemer R, Dobbe JG, Bartels SA, Christiaan BE, Elbers PW, Heger M, Ince C Rapid automatic assessment of microvascular density in sidestream dark field images. Med Biol Eng Comput 2011, 49:1269-1278.

21. Demir SU, Hakimzadeh R, Hargraves RH, Ward KR, Myer EV, Najarian K: An automated method for analysis of microcirculation videos for accurate assessment of tissue perfusion. BMC Med Imaging 2012, 12:37.

22. De BD, Ospina-Tascon G, Salgado D, Favory R, Creteur J, Vincent JL: Monitoring the microcirculation in the critically ill patient: current methods and future approaches. Intensive Care Med 2010, 36:1813-1825.

23. Trzeciak S, Dellinger RP, Parrillo JE, Guglielmi M, Bajaj J, Abate NL, Arnold RC, Colilla S, Zanotti S, Hollenberg SM: Early microcirculatory perfusion derangements in patients with severe sepsis and septic shock: relationship to hemodynamics, oxygen transport, and survival. Ann Emerg Med 2007, 49:88-98. 98.

24. Aalkjaer C, Boedtkjer D, Matchkov V: Vasomotion - what is currently thought? Acta Physiol (Oxf) 2011, 202:253-269.

25. Sallisalmi M, Oksala N, Pettila V, Tenhunen J: Evaluation of sublingual microcirculatory blood flow in the critically ill. Acta Anaesthesiol Scand 2012, 56:298-306.

26. Balestra GM, Bezemer R, Boerma EC, Yong ZY, Sjauw KD, Engstrom AE, Koopmans M, Ince C: Improvement of sidestream dark field imaging with an image acquisition stabilizer. BMC Med Imaging 2010, 10:15.

doi:10.1186/1471-2342-14-14

Cite this article as: Petersen et al: Sidestream dark field images of the microcirculation: intra-observer reliability and correlation between two semi-quantitative methods for determining flow. BMC Medical Imaging 2014 14:14.

\section{Submit your next manuscript to BioMed Central and take full advantage of:}

- Convenient online submission

- Thorough peer review

- No space constraints or color figure charges

- Immediate publication on acceptance

- Inclusion in PubMed, CAS, Scopus and Google Scholar

- Research which is freely available for redistribution 